\title{
NOUVELle
}

\section{FoxP2, un gène qui fait parler I'homme et chanter les oiseaux}

Christelle Rochefort
Neurobiologie de l'Apprentissage, de la Mémoire et de la Communication (NAMC), CNRS UMR 8620, Université Paris Sud, Bâtiment 446, 91405 Orsay France.

christelle.rochefort@u-psud.fr
De la famille $K \varepsilon . .$. aux oiseaux chanteurs

Le langage permet l'expression de la pensée et par la même représente une forme de communication unique à l'homme. Si les voies cérébrales impliquées dans son acquisition ont été décrites de façon approfondie, l'étude des mécanismes moléculaires à l'origine du langage est en revanche très limitée. Néanmoins, des recherches de liaison génétique effectuées sur trois générations d'une famille anglaise, connue sous le nom de «famille $K \varepsilon »$, ont récemment ouvert des pistes particulièrement intéressantes quant aux processus neuronaux du langage. En effet, près de la moitié des membres de cette famille présentent des troubles héréditaires du langage qui se traduisent par une difficulté d'élocution liée à des déficits de mouvements séquentiels de la bouche nécessaires à l'articulation de la parole (dyspraxie verbale) ainsi que des carences grammaticales [1]. Les investigations génétiques chez ces patients ont conduit pour la première fois à l'identification d'un gène unique lié à certains troubles du langage: le facteur de transcription FoxP2 (pour forkhead box P2) [2]. Depuis, des études d'imagerie fonctionnelle chez la famille $K \varepsilon$ [3] ainsi que des analyses génétiques d'autres patients présentant des déficits du langage et de la grammaire similaires à ceux de la famille $K \varepsilon$ [4] ont renforcé l'idée selon laquelle FoxP2 jouerait un rôle déterminant dans le développement des structures neurales impliquées dans la parole et le langage. Ainsi, les investigations sur la famille $K \varepsilon$ ont permis d'ouvrir une formidable piste d'étude des mécanismes moléculaires sous-jacents à la fonction du langage.
Afin d'analyser de façon plus approfondie la contribution de FoxP2 dans les mécanismes sensori-moteurs permettant le développement du langage, il est nécessaire de disposer de modèles animaux adaptés. Or, I'apprentissage vocal, qui représente une composante du langage, se retrouve chez certaines espèces animales dont les oiseaux chanteurs. Ces derniers, à l'instar des êtres humains, acquièrent les sons qui constitueront leur chant en imitant ceux émis par leurs aînés. En d'autres termes, ces oiseaux sont capables de modifier des vocalisations innées pour copier ou créer de nouveaux sons. L'utilisation de ce modèle est d'autant plus pertinente que d'autres parallèles entre la parole humaine et le chant d'oiseau ont pu être établis et incluent l'importance d'une période critique précoce permettant l'apprentissage du chant ainsi que des similitudes dans les circuits cérébraux impliqués dans l'apprentissage et la production des sons [5].

FoxP2 et l'apprentissage du chant chez le diamant mandarin

Le laboratoire du Professeur Constance Scharff utilise le diamant mandarin, une espèce d'oiseau chanteur qui possède un chant quasiment invariable à l'âge adulte, pour examiner le rôle de FoxP2 dans l'apprentissage du chant. Les membres de cette équipe ont notamment montré que, chez les mâles juvéniles, l'expression de I'ARNm de FoxP2 augmente significativement pendant la période sensible d'apprentissage vocal dans l'aire $X$, un noyau de la partie antérieure du cerveau aviaire appartenant au circuit cérébral impliqué dans l'apprentissage du chant [6]. Afin de mettre en évidence un éventuel lien causal entre l'expression de FoxP2 dans l'aire $X$ et l'apprentissage vocal chez des diamants mandarins juvéniles, le Pr Scharff et ses collègues ont ensuite modifié expérimentalement le niveau d'expression de FoxP2 dans ce noyau, via une stratégie basée sur l'interférence ARN couplée à l'utilisation d'un vecteur lentiviral. Cette technique, qui n'avait encore jamais été utilisée dans un cerveau aviaire, est basée sur la propriété des ARN interférentiels à provoquer la destruction des ARNm dont la séquence nucléotidique leur est complémentaire, ce qui a pour conséquence de bloquer la production de la protéine correspondante. L'injection d'un lentivirus contenant un ARN interférentiel anti-FoxP2 dans l'aire $X$, au début de la période critique de plasticité vocale, a donc permis une inhibition locale de FoxP2 pendant la totalité de la période d'apprentissage vocal [7]. Les oiseaux ainsi traités présentent des déficits d'apprentissage du chant par rapport à des oiseaux injectés avec un lentivirus contrôle ne reconnaissant aucun gène aviaire (Figure 1). En effet, chez les oiseaux présentant une diminution de FoxP2, l'imitation du chant du tuteur est non seulement incomplète, mais également imparfaite, puisque la copie des syllabes est souvent inexacte (Figure 1). De plus, ces oiseaux présentent également un chant relativement instable à l'âge adulte [7].

\section{Un rôle de FoxP2 à l'âge adulte?}

Cette étude a donc mis en évidence que l'expression de FoxP2 est critique pour l'acquisition correcte du chant. De plus, sa fonction ne semble pas limitée à la seule mise en place des circuits céré- 
A

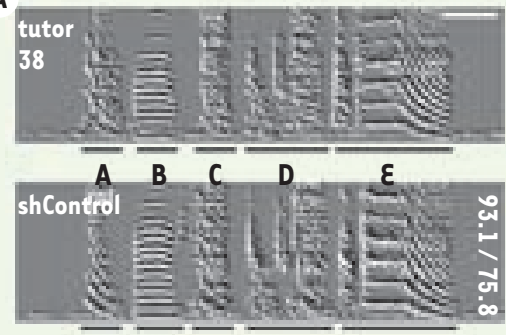

A $\bar{B}$ C
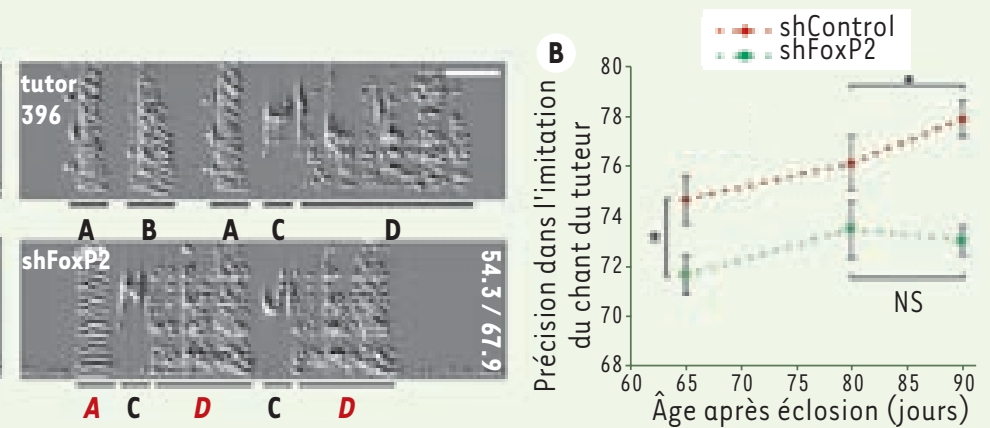

Figure 1. Défauts d'apprentissage du chant chez des oiseaux juvéniles dont l'expression de FoxP2 est bloquée dans l'aire X. A. Sonogrammes représentant le motif chanté par des diamants mandarins, tuteurs (en haut) ou «élèves » (en bas). Chaque motif est composé d’une suite de syllabes, soulignées en noir et codées par des lettres. Les juvéniles déficients en FoxP2 (shFoxP2) apprennent le motif du tuteur de manière incomplète et inexacte : certaines syllabes ne sont pas copiées (syllabe B) et d'autres sont imitées de façon peu précise (lettres rouges en italique). B. Graphique illustrant le score de précision de l'imitation, par les juvéniles, du chant de leur tuteur. Chez les élèves shFoxP2, le déficit d'imitation du chant est visible tout au long de la période d'apprentissage vocal. NS : non significatif. ${ }^{*} p<0,05$ (adapté de [7]).

braux au cours du développement dans la mesure où la diminution expérimentale de FoxP2 n'a été déclenchée qu'à partir de la troisième semaine post-natale. Par conséquent, ces données offrent une nouvelle possibilité d'interprétation des troubles du langage chez les individus portant une mutation de FoxP2, associés jusqu'à présent à des déficits de développement embryonnaire [3, 8]. De manière intéressante, il a été montré que FoxP2 continue d'être exprimé et régulé chez le diamant mandarin et le canari adultes, lors du comportement de chant $[6,9]$. L'hypothèse la plus probable serait donc que, outre le rôle de FoxP2 dans le développement embryonnaire de circuits cérébraux sous-jacents au langage, ce gène soit également impliqué à un stade plus tardif dans les aptitudes sensori-motrices. Toutefois, les travaux de l'équipe du Pr. Scharff ne permettent pas de préciser le rôle postnatal de FoxP2. II reste notamment à distinguer si FoxP2 est important pour la production motrice ou pour l'apprentissage moteur. Des expériences d'inhibition de FoxP2 à l'âge adulte, une fois la période critique d'apprentissage révolue, pourront certainement permettre d'établir une distinction entre ces deux processus. $\diamond$ The FoxP2 gene makes humans speak... and birds twitter

\section{RÉFÉRENCES}

1. Vargha-Khadem F, Gadian DG, Copp A, Mishkin M. FOXP2 and the neuroanatomy of speech and language. Nat Rev Neurosci 2005 ; 6 : 131-8.
2. Lai CS, Fisher SE, Hurst JA, et al. A forkhead-domain gene is mutated in a severe speech and language disorder. Nature $2001 ; 413$ : 519-23.

3. Liégeois F, Baldeweg T, Connelly A, et al. Language fMRI abnormalities associated with FOXP2 gene mutation. Nat Neurosci $2003 ; 6: 1230-7$.

4. MacDermot KD, Bonora $\varepsilon$, Sykes $M$, et al. Identification of FOXP2 truncation as a novel cause of developmental speech and language deficits. Am J Hum Genet $2005 ; 76$ : 1074-80.

5. Doupe AJ, Kuhl PK. Birdsong and human speech : common themes and mechanisms. Annu Rev Neurosci $1999 ; 22: 567-631$.

6. Haesler S, Wada K, Nshdejan A, et al. FoxP2 expression in avian vocal learners and non-learners. J Neurosci $2004 ; 24: 3164-75$.

7. Haesler S, Rochefort C, Georgi B, et al. Incomplete and inaccurate vocal imitation after knockdown of FoxP2 in songbird basal ganglia nucleus Area X. PLoS Biol 2007 ; 5 : e 321.

8. Watkins KE, Dronkers NF, Vargha-Khadem F. Behavioural analysis of an inherited speech and language disorder : comparison with acquired aphasia. Brain 2002 ; 125 : 452-64.

9. Teramitsu I, White SA. FoxP2 regulation during undirected singing in adult songbirds. J Neurosci $2006 ; 26: 7390-4$.

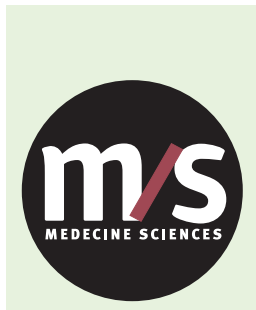

Tarifs d'abonnement M/S - 2008

Abonnez-vous

à Médecine/Sciences
$>$ Grâce à $m / s$, vous vivez en direct les progrès des sciences biologiques et médicales

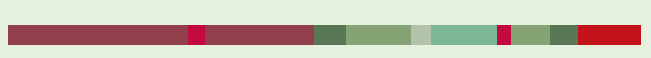

$$
\begin{aligned}
& \text { Bulletin d'abonnement } \\
& \text { page } 928 \text { dans ce numéro de } \mathrm{m} / \mathrm{s}
\end{aligned}
$$

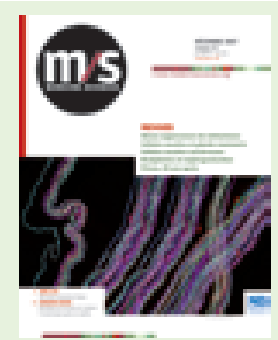

